\title{
Research Note \\ The orbital modulation of the X-ray binary V Sagittae in the high and low states
}

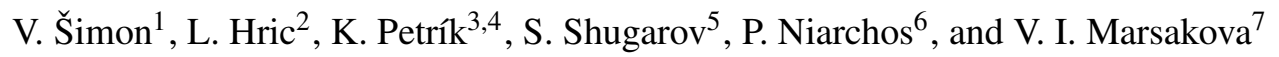 \\ 1 Astronomical Institute, Academy of Sciences of the Czech Republic, 25165 Ondřejov, Czech Republic \\ 2 Astronomical Institute of the Slovak Academy of Sciences, 05960 Tatranská Lomnica, Slovakia \\ ${ }^{3}$ Department of Mathematics, Informatics and Physics, Faculty of Education, University of Trnava, 91843 Trnava, Slovakia \\ ${ }^{4}$ Hlohovec Observatory, Sládkovičova 41, 92001 Hlohovec, Slovakia \\ 5 Sternberg Astronomical Institute, Moscow State University, Universitetsky Prospect 13, 119899 Moscow, Russia \\ ${ }^{6}$ Department of Astrophysics, Astronomy and Mechanics, Faculty of Physics, University of Athens, 15784 Zografos, Athens, \\ Greece \\ 7 Department of Astronomy, Odessa State University, T.G. Shevchenko park, 65014 Odessa, Ukraine
}

Received 5 February 2002 / Accepted 3 July 2002

\begin{abstract}
Our analysis of the orbital modulation of V Sge, carried out in the intensity scale instead of the previously used magnitude scale, revealed that the full amplitude of the modulation remains almost constant as the intensity of the system rises from the low to the high state. The primary minimum remains very similar, as regards both its depth and width. The secondary minimum tends to slightly lag behind phase 0.5 . The depth of the secondary minimum is subjected to the largest changes; it becomes almost as deep as the primary minimum during the high state while it is significantly more shallow than the primary one in the low state.
\end{abstract}

Key words. stars: binaries: general - stars: binaries: close - stars: circumstellar matter - stars: novae, cataclysmic variables stars: individual: V Sge

\section{Introduction}

V Sge is a peculiar eclipsing binary $\left(P_{\text {orb }}=0.514 \mathrm{~d}\right)($ Herbig et al. 1965 - HPSP). Several lines of evidence have been accumulated that strongly support the model in which the white dwarf (WD) primary accretes matter from a massive companion (Patterson et al. 1998; Greiner \& van Teeseling 1998; Śimon \& Mattei 1999). V Sge is therefore a promising candidate for a Galactic counterpart of the super-soft X-ray sources (SSXS) (Steiner \& Diaz 1998). V Sge displays strong longterm photometric activity (e.g. HPSP; Robertson et al. 1997; Šimon \& Mattei 1999).

The orbital modulation of V Sge displays primary and secondary minima (HPSP); the depth of the primary minimum of V Sge dramatically decreases from $1.2 \mathrm{mag}$ in the low state (LS) to less than $0.3 \mathrm{mag}$ in the high state (HS). Uneclipsed light, possibly a circumbinary envelope formed during HS (HPSP; Greiner \& van Teeseling 1998) is supposed to be the main cause of the variations of the orbital modulation.

Previously, the complete coverage of the modulation was achieved by a vertical shifting of the segments (the individual

Send offprint requests to: V. Šimon, e-mail: simon@asu.cas.cz night series) on the magnitude scale (HPSP). This approach was successful if the differences of brightnesses between the series were small (a few tenths of mag). However, Patterson et al. (1998) argued that the depth of the primary minimum remains about the same in the intensity units when the modulation in HS and LS is compared. It should therefore be more advantageous to analyze the modulation in intensities.

A preliminary version of this analysis was presented by Šimon et al. (2002).

\section{Collection of the data}

The observations presented here were obtained at several observatories. The journal of the observations is given in Table 1. The series of the $V$ band measurements, long enough to resolve the orbital modulation, were used for the following analysis. The photoelectric observations were secured by the $600 / 7500 \mathrm{~mm}$ Cassegrain at the Crimea Observatory (JulyNovember 1995, July 1996), 700/10500 mm Cassegrain at the Moscow Observatory of the Sternberg Astronomical Institute (August 1997), 1200/18000 mm Cassegrain at the Kryonerion Astronomical Station of the National Observatory of Athens 
Table 1. Journal of the observations. The respective observatories are listed in the column labeled Obs. and their full abbreviations are given in the footnote to the table. The state of activity of each series, that is group I, II, or III, defined in Sect. 3, is specified. The number of observations in a series is also given. Typical exposure time was 90 or $60 \mathrm{~s}$ for the CCD measurements (Ond. and Ath.) and $10 \mathrm{~s}$ for the photoelectric observations (the remaining observatories). The Julian Date (JD-2 400 000) of the middle of the series is listed in the column labeled JD(mid). The epochs and orbital phases were calculated according to the quadratic ephemeris of Smak (1995). The measurements obtained within 1995-1997 are listed in Šimon et al. (2001).

\begin{tabular}{lllrrc}
\hline \hline Date & $J D($ mid $)$ & Obs. & State & No. & Epoch + phase \\
\hline 14/15 Oct. 94 & 49640.38 & Ond. & $I I I$ & 19 & $22851.914-22852.482$ \\
29/30 Oct. 94 & 49655.29 & Ond. & $I I$ & 16 & $22880.987-22881.387$ \\
27/28 Feb. 96 & 50141.61 & Ska. & $I I$ & 120 & $23826.931-23827.028$ \\
25/26 Oct. 96 & 50382.27 & Ska. & $I I I$ & 16 & $24294.893-24295.152$ \\
28/29 Aug. 97 & 50689.45 & Ska. & $I I I$ & 48 & $24892.379-24892.454$ \\
31 Aug./ Sep. 97 & 50692.43 & Hlo. & $I I I$ & 7 & $24898.144-24898.301$ \\
1/2 Sep. 97 & 50693.53 & Hlo. & $I I I$ & 17 & $24900.329-24900.398$ \\
6/7 Oct. 97 & 50728.39 & Ska. & $I I$ & 184 & $24968.009-24968.304$ \\
19/20 Oct. 98 & 51106.31 & Kry. & $I I$ & 94 & $25702.960-25703.285$ \\
20/21 Oct. 98 & 51107.29 & Kry. & $I I$ & 60 & $25704.934-25705.155$ \\
22/23 Aug. 99 & 51413.44 & Ond. & $I$ & 34 & $26300.241-26300.660$ \\
1/2 Oct. 99 & 51453.39 & Ond. & $I$ & 55 & $26378.010-26378.244$ \\
9/10 Oct. 99 & 51461.38 & Kry. & $I I$ & 26 & $26393.629-26393.731$ \\
11/12 Oct. 99 & 51463.34 & Kry. & $I I$ & 62 & $26397.389-26397.582$ \\
12/13 Oct. 99 & 51464.33 & Kry. & $I I$ & 107 & $26399.248-26399.561$ \\
12/13 Oct. 99 & 51464.36 & Ond. & $I I$ & 52 & $26399.302-26399.640$ \\
13/14 Oct. 99 & 51465.35 & Kry. & $I I$ & 40 & $26401.326-26401.472$ \\
11/12 Dec. 99 & 51524.22 & Ond. & $I$ & 43 & $26515.799-26515.968$ \\
4/5 Sep. 00 & 51792.44 & Kry. & $I I$ & 110 & $27037.305-27037.745$ \\
28/29 Sep. 00 & 51816.35 & Kry. & $I I$ & 115 & $27083.816-27084.219$ \\
12/13 Oct. 00 & 51830.41 & Ath. & $I I$ & 32 & $27111.249-27111.495$ \\
23/24 May 01 & 52053.47 & Ska. & $I I$ & 71 & $27545.043-27545.300$ \\
15/16 Aug. 01 & 52137.47 & Ond. & $I I$ & 30 & $27708.302-27708.762$ \\
\hline
\end{tabular}

Kry...Kryonerion Obs.; Hlo...Hlohovec Obs.; Ska...Skalnaté Pleso Obs.; Ond...Astr. Inst. Ondřejov; Ath...Athens Univ. Obs.

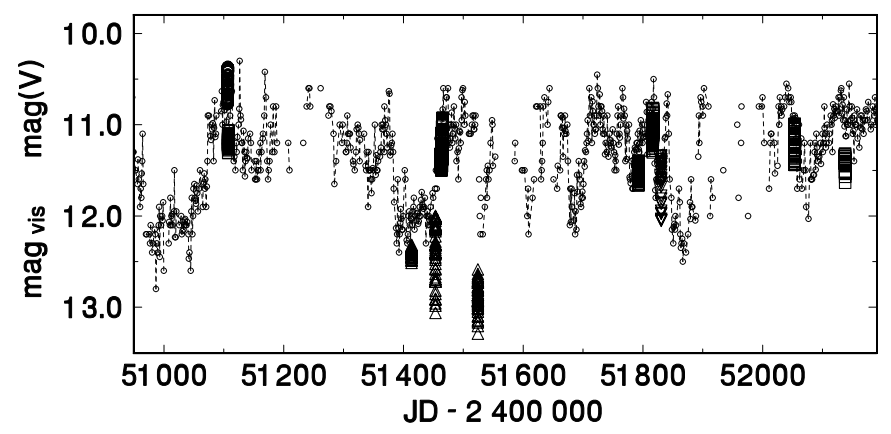

Fig. 1. An illustration of the states of activity of V Sge during which the observations of the orbital modulation were obtained (the measurements obtained within 1995-1997 can be seen in Fig. 1 of Šimon et al. 2001). The data were divided into group I (triangles), group II (squares), and group III (circles). The dots represent the daily means of the out-eclipse visual observations. See Sects. 2 and 3 for details.

(October 1998-October 2000), 600/7500 mm Cassegrain at the Hlohovec Observatory (August 1997-July 1999), $600 / 7500 \mathrm{~mm}$ Cassegrain at the Skalnaté Pleso Observatory (October 1995-May 2001). V-filter CCD images were obtained by Maksutov $180 / 1000 \mathrm{~mm}$, SBIG ST-6 in the Astronomical Institute in Ondřejov during 1999 and 2001 and by 400/1200 mm Cassegrain, CCT-16, DFM Engineering, Inc. at the Athens Observatory in October 2000. Two additional series of unfiltered CCD images (maximum sensitivity in the $R$ band) were obtained in the Astr. Inst. in Ondřejov in October 1994.

V Sge has an optical companion $(V=14.34,9.7 \operatorname{arcsec}$ distant) (HPSP). The measurements at the Crimea and Moscow Observatories were done with a sufficiently small diameter of the diaphragm, in other cases the brightness of V Sge was corrected by calculation. Only the October 1994 series was an exception in this sense because the brightness of the companion could not be determined. Fortunately, a comparison with the visual long-term AAVSO light curve (Mattei 1996) confirmed that the measurements were obtained in HS, around 10.5 mag $(V)$, where the contribution of this star is very small and can be neglected.

The star USNO-A2.0 1050-17330568 was used as the comparison star at all observatories. USNO-A2.0 1050-17224337 served as the check star at the Crimea and Moscow observatories while USNO-A2.0 1050-17274516 was used at the Skalnaté Pleso and Hlohovec observatories. USNO-A2.0 1050-17287011 was used as the check star for the Ondřejov and Athens observations.

In order to illustrate which kind of activity our observations represent, the visual light curve is superimposed in Fig. 1. It consists of the daily means of the out-eclipse observations 

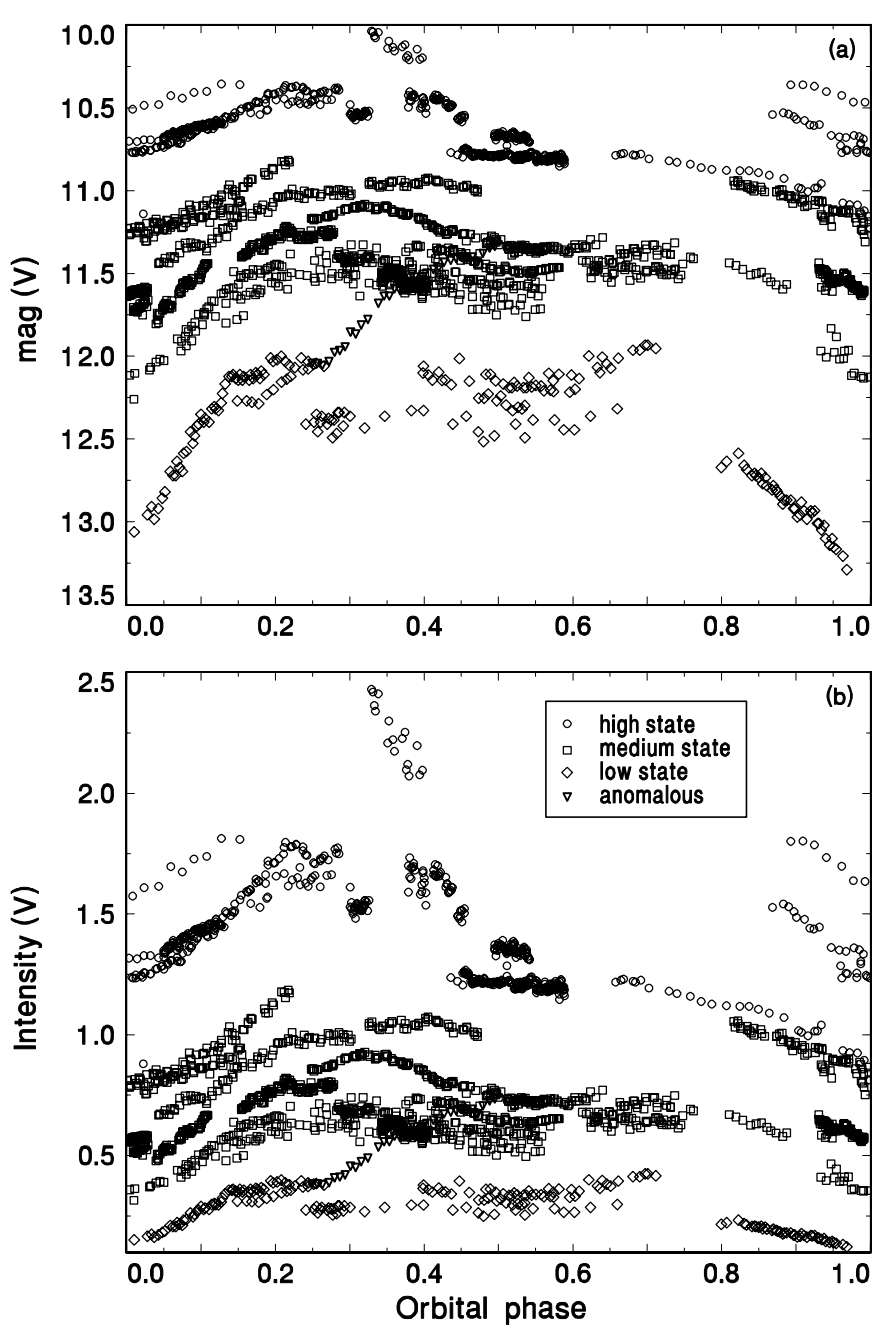

Fig. 2. All observations of V Sge in magnitude a) and intensity b) scale, folded with the orbital phase. The sorting into groups I, II, and III is marked. The "anomalous" behaviour refers to the series in JD 2451 830. See Sect. 3 for details.

(phases 0.1-0.9). The visual data come from the database of AFOEV, operated at CDS, France, and VSNET, Japan.

\section{Data analysis}

We carried out a similar procedure as HPSP, but now on the intensity scale. All the $V$ band observations were transformed into intensity units, setting the intensity equal to unity at $11 \mathrm{mag}(V)$. This value roughly corresponds to the mean magnitude of the long-term light curve. All the observations, folded with the orbital phase, are shown in Figs. 2a, b. The data in both the magnitude and intensity scale are shown for comparison. The quadratic ephemeris of Smak (1995) was used for the calculation of the orbital phases:

$$
\begin{aligned}
T(\operatorname{Min} I)= & 2437889.9157+0^{\mathrm{d}} .51419706 E \\
& -1.23 \times 10^{-10} E^{2} .
\end{aligned}
$$

In most nights, the modulation in V Sge is clearly detectable and the series display the common course of modulation, albeit with a shift in intensity. In order to make the assessment
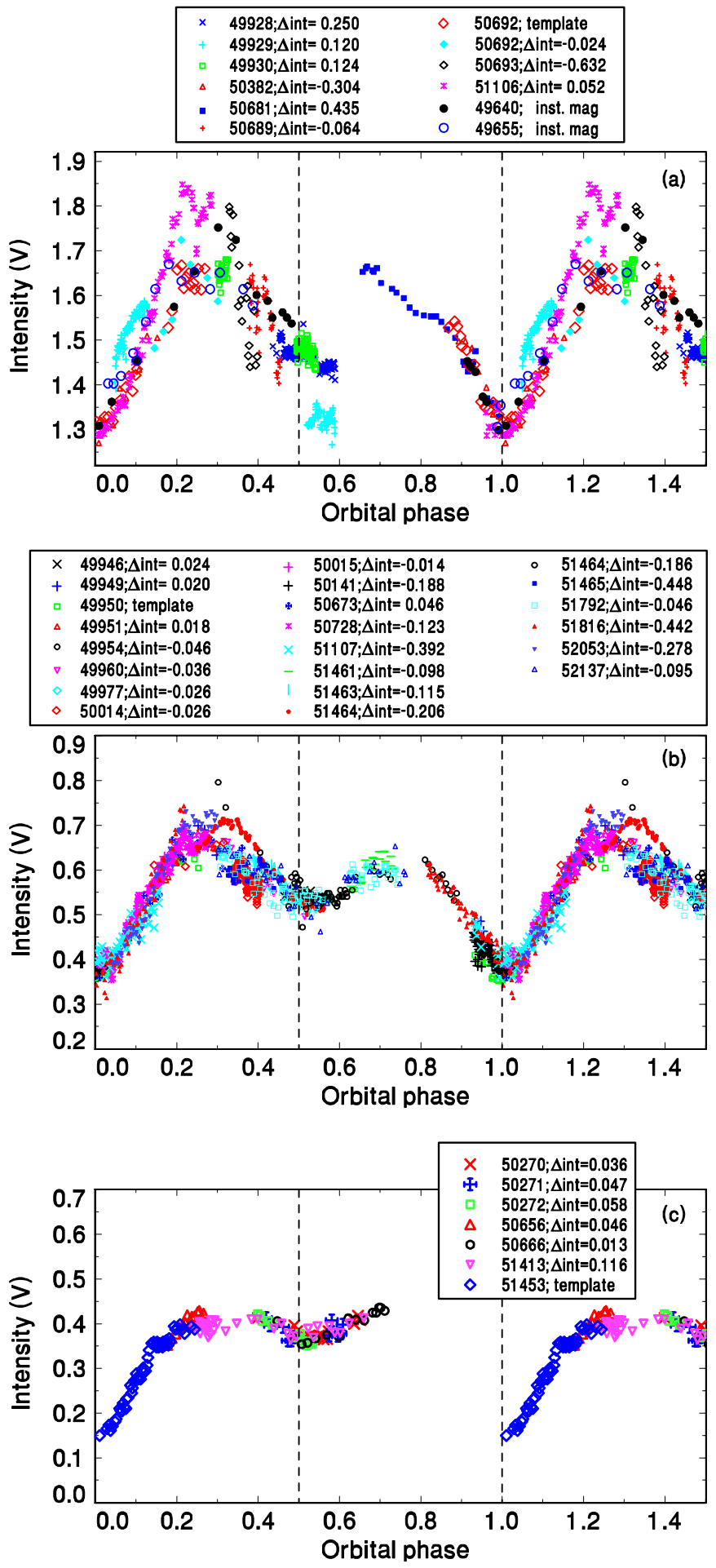

Fig. 3. The observations from Fig. 2b, sorted into three groups according to their intensity. The individual curves of each group were shifted in intensity to match the template. The templates for each group are marked in the legends. The scale of the ordinate is identical for all three plots. See Sect. 3 for details. (This figure is available in color in electronic form.)

of the changes from the individual night series tractable, the observations from Fig. 2 were sorted into three groups according to their intensity: group I $(I \leq 0.4)$, group II $(0.4<I \leq$ $1.1)$, group III $(1.1<I)$. The intensity of the whole series, 
sometimes apart from the bottom of the primary eclipse, lies inside the interval relevant for the given group. This sorting was proven to reflect the changes of the course of the modulation with increasing intensity in most cases. In addition, these three groups are related to the states of the optical activity of V Sge (see below). One well-covered and long series was chosen for each group and served as a template (see Fig. 3). The remaining series were interactively matched to this template using the code CORFOT, developed by one of us. No template covered the whole orbital light curve because of the long $P_{\text {orb }}$ and relatively short nights during the seasonal visibility of V Sge. We tried not to minimize artificially the scatter at any particular phase. The resulting curves are shown in Figs. 3a, b, c. The modulation in the respective orbital phases is not uniformly covered due to the fact that the extreme cases (groups I and III) occurred more rarely than group II during the years in which our observations were obtained.

Group I and III can be associated with LS and HS, respectively. Group II may be compared to a medium state (MS), called flat segment by Šimon \& Mattei (1999). An episode of MS occurred within JD 2449 940- 2450190 (see Šimon et al. 2001 for more). Naturally, group II contains also the transitions between HS and LS. The level of HS tended to decrease during the season in which our data were secured, therefore some series fall more often to group II instead of III. In this respect, group III may possibly be called an "extreme" HS.

The amplitude of the orbital modulation of V Sge in the intensity scale remains almost the same as the intensity of the system rises. The shape of the primary minimum remains similar, as regards both its depth and width. On the contrary, the depth of the secondary minimum is subjected to the largest changes. It becomes very deep when V Sge is bright (group III) while it is just barely detectable when the system is faint (group I). Also the profile around phase 0.25 undergoes large changes (the coverage around phase 0.75 was often poor). Such a behaviour is quite different from that seen in the magnitude scale.

The phase of the secondary minimum for group I and II could be determined by fitting the polynomials. This yielded phase 0.525 and 0.540 , respectively, the uncertainty being $\sim 0.01$ phase. We also checked the phase of the primary minimum of group II and III and found that they slightly lag by 0.01 phase, with the uncertainty of $\sim 0.01$ phase. This shift of the primary minimum may be caused by the composition of many curves, which introduce a scatter, and/or by a slight deviation of $P_{\text {orb }}$ from the ephemeris. In summary, the lag of the secondary minimum is small in our data. Nevertheless, this lag is in the same sense as that observed in the curves by Smak et al. (2001, their Fig. 6).

\section{Discussion}

Our analysis of the orbital modulation of V Sge in the intensity scale revealed large changes of its profile between the HS and LS, particularly around phase 0.5 . First, we will discuss the model-independent conclusions.

The stability of the amplitude of the modulation through LS to HS is not trivial, given the large change of its profile. The flat profile outside the primary eclipse in LS can be interpreted in terms of the bulk luminosity originating from the primary, without strong proximity effects (reflection effect and ellipticity). More generally, there are no signs of deviation from the symmetry of the primary (bulges, hot or cool spots, asymmetric outflows) in the continuum. The reflection effect in LS is negligible although it should be prominent; the large secondary $\left(q=M_{2} / M_{1}=3.8\right.$; HPSP $)$ is significantly darker than the primary and should act as a reflecting screen. This effect is absent also in UV in LS (Wood \& Lockley 2000). The rounded profile outside the primary eclipse in MS and HS suggests that these effects may become apparent. The difference between the first and the second quadrature in MS implies that a strongly asymmetric distribution of brightness with respect to the line joining the stars appears. This modulation is similar to that in the so called "flat segment B" of Šimon \& Mattei (2000). The large depth of the secondary minimum in HS in the "classical" interpretation implies that the temperatures of both components, $T_{\text {eff }}$, are not very different. In this case, a decrease of the amplitude in HS with respect to that in LS would be expected because of a more shallow primary eclipse. It therefore appears that the respective effects conspire to produce almost unchanged amplitude in all states.

The anomalous modulation observed in JD 2451830 and possibly also JD 2450681 must be quite rare because otherwise we would not be able to resolve the orbital modulation in V Sge at all. This anomaly was always observed in the phase interval when the disk is most exposed to the observer. The amplitude of the rise in JD 2451830 (at least $0.5 \mathrm{mag}(V)$ ) is larger than the rate of rise over a comparable time interval during a LS/HS transition (Šimon \& Mattei 1999). It can be inferred from Fig. 1 that the duration of this event was of the order of a single orbital cycle.

The scatter of the respective composed light curves, increasing through LS to HS, can be attributed mainly to cycleto-cycle fluctuations of brightness of V Sge. If their amplitude remains stable on the magnitude scale rather than the intensity scale then a larger scatter for HS will emerge in Fig. 3.

In the following, we will make use of the model for V Sge in which the WD primary accretes matter from a more massive secondary (donor) (see Sect. 1). The physics of irradiated accretion disks and mass transfer variations in such a system offer a more plausible explanation, especially for the long-term large amplitude activity of V Sge, than the alternative nearly-contact model by e.g. HPSP and Smak et al. (2001). A discussion of both models, which favours the WD model, was presented by Šimon \& Mattei (1999). The modulation of V Sge in LS is quite similar to the SSX binary CAL 87 and hence the interpretation by the model of Schandl et al. (1997) also appears viable, as stressed by Patterson et al. (1998). On the contrary, Smak et al. (2001) attempted to fit just a model with a standard disk which failed also for CAL 87 (Schandl et al. 1997). However, we note that the behaviour of the spectral lines, revealed by Wood \& Lockley (2000) and interpreted in terms of the colliding-wind binary may not be in contradiction with the WD model. Van den Heuvel et al. (1992) suggested a model for the SSX binary CAL 87 in which the irradiation of the donor invokes its wind which then collides with the wind from the WD 
and the disk (see also van Teeseling \& King 1998 for a general model). A similar scenario may apply also to V Sge.

The flat profile of the orbital modulation in LS can be explained if the disk is symmetric and the donor is partly shielded by the disk so that the reflection effect is diminished. The rounded profile and unequal height of the first and the second quadrature in MS can be obtained in the framework of the model of Meyer-Hofmeister et al. (1997) if the elevated rim (bulge), invoked by the stream impact onto the disk, appears when the mass transfer rate increases to $\dot{m} \geq 10^{-7} M_{\odot} \mathrm{yr}^{-1}$. At phase 0.25 we look at the inner side of the bulge which is irradiated by the WD while at phase 0.75 we see just the outer, non-irradiated side. This bulge can also account for the delay of the secondary minimum because the profile of the modulation strongly depends on the shape of the bulge (Meyer-Hofmeister et al. 1997).

The appearance of the deep secondary minimum in HS is difficult to explain by any model for V Sge. A very large intrinsic increase of $T_{\text {eff }}$ of the donor by several tens of thousands of Kelvins appears rather unphysical; for example the shape of the long-term light curve (e.g. Šimon \& Mattei 1999) does not resemble anything like pulsations. The secondary minimum resulting just from the occultation of the side of the donor heated by the disk is significantly more shallow than the primary one (see Meyer-Hofmeister et al. 1997), unless a large amount of heat transport beyond the irradiated hemisphere is introduced. As an alternative approach, we may assume that the disk luminosity always dominates and that the shape of the disk becomes very asymmetric in HS, with a vertically and azimuthally extended bulge. The model by Meyer-Hofmeister et al. (1997) shows that the bulge becomes an important source of highly modulated light. The middle model curve in their Fig. 6 shows that such a disk can give rise to the modulation with two deep minima and rounded maxima.
Acknowledgements. This research has made use of NASA's Astrophysics Data System Abstract Service, the AFOEV database, operated at CDS, France, VSNET, Japan, and AAVSO (Massachusetts, USA). The support by the project ESA PRODEX INTEGRAL 14527 is acknowledged. The research of V.Š. is supported by the post-doctoral grant 205/00/P013 of the Grant Agency of the Czech Republic. The research of L.H. is supported by the VEGA grant $1008 / 21$ of the Slovak Republic. The research of K.P. is supported by the VEGA grant 1008/21 of the Slovak Republic and by the internal grant of the Faculty of Education No. 1/98. We thank Ms I. Stoklasová for reducing some of the Ondřejov data.

\section{References}

Greiner, J., \& van Teeseling, A. 1998, A\&A, 339, L21

Herbig, G. H., Preston, G. W., Smak, J., \& Paczynski, B. 1965, ApJ, 141, 617 (HPSP)

Meyer-Hofmeister, E., Schandl, S., \& Meyer, F. 1997, A\&A, 321, 245

Mattei, J. 1996, AAVSO International database, private communication

Patterson, J., Kemp, J., Shambrook, A., et al. 1998, PASP, 110, 380

Robertson, J. W., Honeycutt, R. K., \& Pier, J. R. 1997, AJ, 113, 787

Schandl, S., Meyer-Hofmeister, E., \& Meyer, F. 1997, A\&A, 318, 73

Smak, J. 1995, Acta Astron., 45, 361

Smak, J., Belczynski, K., \& Zola, S. 2001, Acta Astron., 51, 117

Steiner, J. E., \& Diaz, M. P. 1998, PASP, 110, 276

Šimon, V., \& Mattei, J. A. 1999, A\&AS, 139, 75

Šimon, V. \& Mattei, J. A. 2000, Ap\&SS, 272, 333

Šimon, V., Shugarov, S., \& Marsakova, V. I. 2001, A\&A, 366, 100

Šimon, V., Hric, L., Petrík, K., et al. 2002, The Physics of Cataclysmic Variables and Related Objects, ed. B. T. Gansicke, K. Beuermann, \& K. Reinsch, ASP Conf. Proc., 261, 663

van den Heuvel, E. P. J., Bhattacharya, D., Nomoto, K., \& Rappaport, S. A. 1992, A\&A, 262, 97

van Teeseling, A., \& King, A. R. 1998, A\&A, 338 , 957

Wood, J. H., \& Lockley, J. J. 2000, MNRAS, 313, 789 